\title{
Estimation of the Relaxation Factor for Small Mesh Size
}

Recently Frankel [1] and Young [3, 4] have developed a method of successive overrelaxation for the solution of the difference equation analogue of the Dirichlet problem for a linear elliptic partial differential equation. The purpose of this note is to present a simple asymptotic theory of the overrelaxation process which depends on the assumption that the mesh size $h$ approaches zero, but which describes adequately the practical aspects of the earlier work. Thus the results discussed are not essentially new, but it is hoped that our approach to them will provide an easy insight into the overrelaxation scheme for those interested in its application to specific examples.

We consider the Laplace difference equations for an unknown function $u$ of two independent variables in a region $D$ covered by net points spaced $h$ units apart. In a standard notation, we use subscripts $i$ and $j$ to refer to the location of net points in our grid and we use the superscript $n$ to indicate steps in the relaxation process, so that, in its simplest form, the method of successive overrelaxation can be described by the equation

$$
4\left(u_{i, j}^{n+1}-u_{i, j}^{n}\right)=r\left(u_{i-1, j}^{n+1}+u_{i, j-1}^{n+1}+u_{i+1, j}^{n}+u_{i, j+1}^{n}-4 u_{i, j}^{n}\right),
$$

where $r$ is the relaxation factor. We express $r$ in the form

$$
r=\frac{2}{1+C h}
$$

for any positive value of the constant $C$, and we rearrange (1) to obtain

$$
\begin{array}{r}
\frac{u_{i-1, j}^{n}+u_{i, j-1}^{n}+u_{i+1, j}^{n}+u_{i, j+1}^{n}-4 u_{i, j}^{n}}{h^{2}}=\frac{u_{i, j}^{n+1}-u_{i, j}^{n}-u_{i-1, j}^{n+1}+u_{i-1, j}^{n}}{h^{2}} \\
+\frac{u_{i, j}^{n+1}-u_{i, j}^{n}-u_{i, j-1}^{n+1}+u_{i, j-1}^{n}}{h^{2}}+2 C \frac{u_{i, j}^{n+1}-u_{i, j}^{n}}{h}
\end{array}
$$

Using the familiar idea that the index $n$ refers to a new time variable, $t$, and that it indicates the location of new net points spaced at time intervals equal to the original mesh size $h$, we recognize that (3) is the difference analogue of the hyperbolic partial differential equation

$$
u_{x x}+u_{y y}=u_{x t}+u_{y t}+2 C u_{t}
$$

Thus for small values of $h$ the convergence of the iterative scheme (1) can be investigated by an analysis of the decay of time-dependent terms in the solution of (4). 
The substitution $s=t+x / 2+y / 2$ brings (4) into the canonical form

$$
u_{x x}+u_{y y}-\frac{1}{2} u_{s s}-2 C u_{s}=0 .
$$

For a fixed set of boundary conditions, the method of separation of variables yields the representation

$$
u=U_{0}(x, y)+\sum_{m=1}^{\infty}\left[a_{m} \exp \left(-p_{m} s\right)+b_{m} \exp \left(-q_{m} s\right)\right] U_{m}(x, y)
$$

for the solution $u$ of (5), where $U_{0}$ is the steady-state solution, where the $a_{m}$ and $b_{m}$ are Fourier coefficients, where

$$
p_{m}=2 C-\left(4 C^{2}-2 k_{m}^{2}\right)^{\frac{1}{2}}, \quad q_{m}=2 C+\left(4 C^{2}-2 k_{m}^{2}\right)^{\frac{1}{2}},
$$

and where the $U_{m}$ and $k_{m}{ }^{2}$ are the eigenfunctions and eigenvalues of the problem

$$
\Delta U_{m}+k_{m}^{2} U_{m}=0
$$

with homogeneous boundary conditions. The exponent

$$
p=\operatorname{Re}\left[p_{1}\right]=\operatorname{Re}\left[2 C-\left(4 C^{2}-2 k_{1}^{2}\right)^{\frac{1}{2}}\right]
$$

corresponding to the lowest eigenvalue $k_{1}{ }^{2}$ governs the rate of decay of the terms on the right in (6) with increasing time $t$. Thus the error $E$ in the solution of the Laplace difference equations obtained through $N$ iterations of the overrelaxation scheme (1) has the order of magnitude

$$
E=O\left(e^{-p N h}\right),
$$

since it is approximated by the infinite series used in the representation (6) for time $t=N h$. It follows that we must iterate $N=O(1 / h)$ times to arrive at a desired degree of accuracy in the solution.

By (9), the choice of the positive constant $C$ which maximizes $p$ and therefore yields the most rapid convergence is clearly $C=k_{1} / 2^{\ddagger}$, and an underestimate of this best value is less damaging than an overestimate (cf. [3]). Indeed, the radical in the expression for $p$ is pure imaginary and can be omitted when $C<k_{1} / 2^{\frac{1}{3}}$, whereas this same radical causes a sharp decline in the value of $p$ when $C$ is taken slightly larger than $k_{1} / 2$. A simple lower bound on the principal frequency $k_{1}$ for the case of Dirichlet boundary conditions is given by Pólya and Szegö [2]. If $A$ denotes the area of the region $D$, they show that

$$
k_{1} A^{\frac{3}{3}} \geq k \pi^{\frac{3}{3}},
$$

where $k=2.405$ denotes the first root of the Bessel function of the first kind of order zero. Thus a good approximate formula for the relaxation factor $r$ is

$$
r=\frac{2}{1+(\pi / 2 A)^{\frac{1}{2} k h}}=\frac{2}{1+3.014 h / A^{\frac{1}{3}}} .
$$


Numerical examples worked out by Young [4] indicate that when (12) is used instead of the exact best value of $r$, the additional iterations required to achieve equivalent accuracy will usually not exceed 20 per cent of the number needed with the optimal $r$.

Our approach shows that the method of successive overrelaxation applies to the nine-point Laplace difference equation

$$
\begin{aligned}
4 u_{i-1, j}+4 u_{i, j-1}+4 u_{i+1, j}+4 u_{i, j+1}+ & u_{i-1, j-1}+u_{i+1, j-1} \\
& +u_{i+1, j+1}+u_{i-1, j+1}-20 u_{i, j}=0
\end{aligned}
$$

It is easily verified that a relaxation factor $r$ of the form (2) leads in the case of (13) to the hyperbolic partial differential equation

$$
3 u_{x x}+3 u_{y y}=2 u_{x t}+3 u_{y t}+5 C u_{t}
$$

similar to (4), and that the best choice for the parameter $C$ here is $C=13^{\frac{1}{k}} k_{1} / 5$, while the corresponding value for the exponent $p$ governing the rate of convergence of the iterations is $p=6 k_{1} / 13^{\frac{1}{3}}$. Thus we see that these quantities are slightly larger for the nine-point equation than they are for the five-point equation, a result not inconsistent with the numerical data compiled by Young [4], which indicated that the optimal value of $r$ decreases and the correspondingly smallest number of required iterations changes insignificantly when we pass from the fivepoint to the nine-point interpolation formula. The strong dependence of the latter formula for the Laplace operator on the particular partial differential equation involved appears to have obscured until now the explanation of this phenomenon. At all events, for the nine-point difference equation the asymptotic expression for the best value of the relaxation factor $r$ is

$$
r=\frac{2}{1+13^{\frac{1}{k}} k_{1} h / 5} \leq \frac{2}{1+3.074 h / A^{\frac{3}{3}}}
$$

Finally, our introduction of the second order hyperbolic differential equation (4) indicates an analogy between the successive overrelaxation scheme and the second order Richardson method, which is based on a more readily accessible hyperbolic equation (cf. [1]) and converges at a comparable speed. The advantage of successive overrelaxation is that it requires retention of less data and is therefore more adaptable for use on high speed computing machines.

Stanford University

P. R. GARABEDIAN

Stanford, California

1. S. Frankel, "Convergence rates of iterative treatments of partial differential equations," $M T A C$, v. 4, 1950, p. 65-75.

2. G. Pólya \& G. Szegö, Isoperimetric Inequalities in Mathematical Physics, Princeton Univ. Press, 1951.

3. DAvId Young, "Iterative methods for solving partial difference equations of elliptic type," Amer. Math. Soc., Trans., v. 75, 1954, p. 92-111.

4. David M. Young, "ORDVAC solutions of the Dirichlet problem," J. Assoc. Computing Machinery, v. 2, 1955, p. 137-161. 